\title{
Fluorescence correlation spectroscopy with sub-diffraction-limited resolution using near-field optical probes
}

\author{
Dusan Vobornik, ${ }^{1}$ Daniel S. Banks, ${ }^{2}$ Zhengfang Lu, ${ }^{1}$ Cécile Fradin, ${ }^{2, a)}$ Rod Taylor, ${ }^{3, a)}$ and \\ Linda J. Johnston ${ }^{1, a)}$ \\ ${ }^{1}$ Steacie Institute for Molecular Sciences, National Research Council of Canada, Ottawa, Ontario KIA OR6, \\ Canada \\ ${ }_{3}^{2}$ Department of Physics and Astronomy, McMaster University, Hamilton, Ontario L8S 4M1, Canada \\ ${ }^{3}$ Institute for Microstructural Sciences, National Research Council of Canada, Ottawa, Ontario K1A OR6, \\ Canada
}

(Received 10 May 2008; accepted 20 September 2008; published online 24 October 2008)

\begin{abstract}
We report fluorescence correlation spectroscopy (FCS) measurements using near-field scanning optical microscopy (NSOM) probes to produce a sub-diffraction-limited observation area. An order of magnitude reduction in the area compared to confocal FCS has been achieved. We also demonstrate a simple means to model the autocorrelation decay due to diffusion within the excitation profile at the NSOM probe aperture. The use of probes with smaller apertures is expected to provide an additional order of magnitude reduction in the observation area, thus enabling the study of cellular membranes with higher concentrations of fluorophores than is currently possible with diffraction-limited techniques. (C) 2008 American Institute of Physics.
\end{abstract}

[DOI: 10.1063/1.2998602]

Various optical techniques have been recently developed with sufficient sensitivity to detect single molecules. Conventional fluorescence correlation spectroscopy (FCS) typically uses the diffraction-limited optics of confocal microscopy to probe single-molecule dynamics in fluid observation volumes as low as $0.1 \mathrm{fl}$ or on membrane surface areas as low as $\sim 0.1 \mu^{2}$. Since FCS is based on temporal analysis of fluctuations in a fluorescence signal due to individual molecules, it is suited to low particle numbers in the observation volume, which corresponds to nanomolar concentrations of the fluorescent species for diffraction-limited optics. Recently, efforts have been made to observe smaller spatial scales with FCS in order to observe samples with higher concentrations. These methods include stimulated emission depletion, which has achieved a five times reduction in the observation volume, ${ }^{1}$ the application of a supercritical angle objective, which reduces the observation volume in the axial direction at the cost of an increase in the radial direction, ${ }^{2}$ the application of arrays of sub-wavelength-sized apertures [often referred to as zero-mode wave-guides (ZMW)] in thin films which produce attoliter ${ }^{3,4}$ and even zeptoliter observation volumes (or observation areas in lipid membranes of $\left.0.002-0.03 \mu \mathrm{m}^{2}\right),{ }^{5,6}$ and total internal reflection FCS to reduce the excitation volume along the optical axis. ${ }^{7}$ Here, we report the combination of FCS with probes used for nearfield scanning optical microscopy (NSOM) to achieve subdiffraction-limited observation areas. We also demonstrate a simple means to model the autocorrelation decay due to diffusion within the excitation profile at the NSOM tip.

NSOM can achieve the same observation length scales as ZMWs. However, NSOM is more suitable for many applications with membranes since the NSOM probe can be moved to various locations on the membrane for sequential

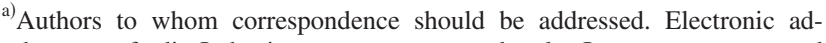
dresses: fradin@physics.mcmaster.ca, rod.taylor@nrc-cnrc.gc.ca, and linda.johnston@nrc-cnrc.gc.ca.
}

measurements. Because of the simplicity of the observation geometry, we are able to demonstrate here that FCS-NSOM data may be analyzed using a simple analytical model in contrast to FCS in ZMWs that significantly perturb the shape of the membrane and require the use of nonanalytical functions to model the data. ${ }^{6}$

Our NSOM experiments use bent, aluminum-coated, and etched optical fiber probes to illuminate a sub-diffractionlimited volume immediately below an aperture created by nanomachining with a focused ion beam. In our NSOM apparatus, which is described elsewhere, ${ }^{8}$ the fluorescence is collected by a 0.75 numerical aperture (NA) objective below the sample, detected by an avalanche photodiode, and correlated by a multitau correlator.

As a proof of the FCS-NSOM principle, we measured diffusion of Oregon Green 1,2-dihexadecanoyl-sn-glycero-3phosphoethanolamine (OG-DHPE) in a dioleoylphosphatidylcholine (DOPC) bilayer on a glass cover slip (see cartoon in Fig. 1 and supplemental material ${ }^{9}$ ). The NSOM tip aperture diameter was $140 \mathrm{~nm}$ as measured by the full width at half maximum of the smallest features observed in a NSOM image of $40 \mathrm{~nm}$ fluorescent beads. Typical correlation data corresponding to the diffusion of OG-DHPE in a planar DOPC bilayer on glass are shown in Fig. 1 for both confocal FCS $(\mathrm{NA}=1.2)$ and FCS-NSOM. The NSOM data shown are representative of data obtained with a number of different probes and for multiple areas of multiple bilayers (see supplemental material). Both FCS and confocal data exhibit a fast correlation decay on the $1-10 \mu$ s time-scale and a slow correlation decay on the millisecond time-scale. The slow correlation decay is attributed to the lateral diffusion of the labeled lipids within the bilayer. The fast correlation decay is attributed to intersystem crossing. We thus model the data by 


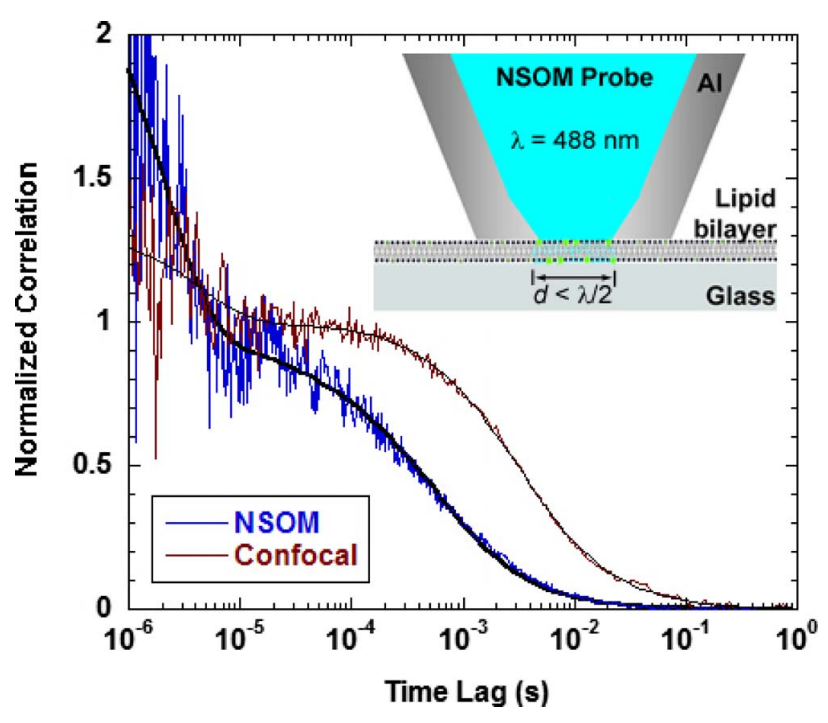

FIG. 1. (Color online) Normalized correlation data for the diffusion of OGDHPE in DOPC lipid bilayers observed with near-field (blue, left curve) and confocal (red, right curve) optics. Both sets of data are fit with Eq. (1) (solid lines). For the confocal data, $G_{D}(t)$ is defined by Eq. (2), which assumes a Gaussian observation area, while for the NSOM data, $G_{D}(t)$ is defined by Eq. (3), which approximates the correlation function for a circular step function observation area. The inset shows a cartoon diagram of the FCSNSOM experiment.

$$
G(\tau)=G_{D}(\tau)\left(1+\frac{T}{1-T} e^{-\tau / \tau_{T}}\right),
$$

where $T$ is the fraction of particles in a nonfluorescent state and $\tau_{T}$ is the characteristic relaxation time of the intersystem crossing. $G_{D}(\tau)$ is the component of the correlation due to diffusion, which in the case of two-dimensional (2D) diffusion with confocal optics is

$$
G_{D}(\tau)=\frac{1}{N}\left(1+\frac{\tau}{\tau_{D}}\right)^{-1}
$$

Equation (2) is calculated assuming that the radial excitation profile is a Gaussian function. $N$ is the average number of fluorophores in the observation volume, and $\tau_{D}=R^{2} / 4 D$ is the characteristic diffusion time, where $R$ is the $1 / e^{2}$-radius of the Gaussian, and $D$ is the fluorophore diffusion coefficient. The confocal FCS data are well described by Eq. (2), indicating a single fluorescent diffusing species. Given $R$ $=190 \mathrm{~nm}$ (as measured from calibration with fluorescein) the fit yields $D=2.8 \pm 0.3 \mu \mathrm{m}^{2} / \mathrm{s}$, which is comparable to a published value for the same system on mica $(D$ $\left.=3.1 \mu \mathrm{m}^{2} / \mathrm{s}\right) .{ }^{10}$ The OG-DHPE surface concentration inferred from the confocal FCS data is $138 \pm 15 \mu \mathrm{m}^{-2}$, in excellent agreement with the expected $140 \mu \mathrm{m}^{-2}$ concentration. However, the FCS-NSOM data shown in Fig. 1 exhibit a broader decay than the confocal data, which is not reflected by Eq. (2). Although the radial excitation intensity profile created by the near-field tip is not well known, it is not expected to be Gaussian. ${ }^{3,11}$ Therefore a different model is needed to describe the correlation data for diffusing particles acquired by FCS-NSOM.

In the FCS-NSOM experiment, the tip is in close contact with the bilayer. We assume that the excitation intensity is constant over the $5 \mathrm{~nm}$ bilayer thickness. Due to the sharply defined circular aperture and possible photosaturation ef-

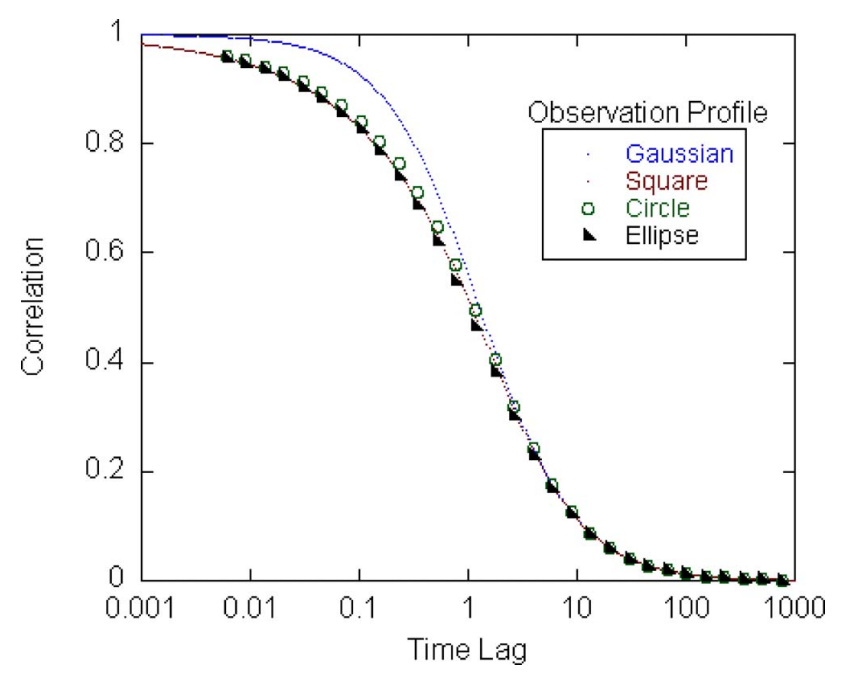

FIG. 2. (Color online) Calculated 2D diffusion correlation function $(N=1)$ for a Gaussian observation profile [Eq.( (2)] and for square [Eq. (3)], circular, and elliptical step function observation profiles. The effective observation area was the same in each case; thus $R=r=(2 / \sqrt{ } \pi) s=a \sqrt{ } 2$ and $b=2 a$, where $R$ is the $1 / e^{2}$-radius of the Gaussian, $r$ is the circle radius, $2 s$ is the side of the square, and $a$ and $b$ are the half-axes of the ellipse. See the supplemental material (Ref. 9) for details.

fects, we hypothesize that there is a uniform probability of detecting a fluorescence photon from a fluorophore directly below the aperture, but the probability of detection decays quite sharply beyond the metallic-glass boundary. Therefore we propose to model the radial observation profile immediately below the tip as a circular step function. The correlation function for $2 \mathrm{D}$ diffusion and a circular step function observation profile were calculated numerically and is shown in Fig. 2. We compared it to the correlation functions for square and elliptical step function observation profiles. Details on the calculation of the correlation functions are given in the supplemental material. ${ }^{9}$

For all three step function profiles, the corresponding correlation functions are significantly stretched at fast-time scales compared to the Gaussian profile. The early correlation decay is attributed to the sharpness of the boundary, which allows fluorophore detection for very short time periods. The same qualitative behavior has previously been simulated for three-dimensional diffusion in cubic and spherical observation volumes. ${ }^{12}$ Although no analytical solution has been found for the circular and elliptical cases, for the square step function observation profile

$$
G_{D}(\tau)=\frac{1}{N}\left[\operatorname{erf}\left(\sqrt{\frac{4 \tau_{D}^{\prime}}{\tau}}\right)-\frac{1}{\sqrt{\pi}} \sqrt{\frac{\tau}{4 \tau_{D}^{\prime}}}\left(1-e^{-4 \tau_{D}^{\prime} / \tau}\right)\right]^{2} .
$$

Here $\operatorname{erf}(\xi)$ denotes the error function and $\tau_{D}^{\prime}=s^{2} / 4 D$ where $s$ is the half-length of a side of the square. Our direct calculation of Eq. (3) is provided in the supplemental material. We note that Eq. (3) is a special case of an autocorrelation function previously derived by Thompson et al. ${ }^{13}$ Equation (3) models well the experimental FCS-NSOM correlation data shown in Fig. 1 and approximates the exact numerical result for the case of the circular observation profile (Fig. 2, the difference in the half-time of the decays is only $2 \%$ ). It has the advantage of ease of use through commercially available curve fitting software. Equation (3) also 
approximates well the case of an elliptical observation profile (Fig. 2), which may result from the polarization of the excitation beam or imperfections in the tip aperture.

Therefore we apply the circular step function model as approximated by Eq. (3) to the FCS-NSOM data. For FCSNSOM, the characteristic diffusion time $\tau$ for the lipid is $380 \pm 40 \mu \mathrm{s}$, an order of magnitude faster than for confocal $(\tau=3.3 \pm 0.3 \mathrm{~ms})$. Using $2.8 \mu \mathrm{m}^{2} / \mathrm{s}$ for the diffusion of OG-DHPE in the bilayer, we calculate the effective radius of the circular step function to be $r=2 s / \pi^{1 / 2}=73 \mathrm{~nm}$, in excellent agreement with our measured value of the tip aperture radius $(r=70 \mathrm{~nm})$. The FCS-NSOM observation area achieved here $\left(0.017 \mu \mathrm{m}^{2}\right)$ is thus approximately an order of magnitude smaller than in the confocal geometry $\left(0.11 \mu \mathrm{m}^{2}\right)$.

Finally, it is of interest to compare the efficiency of FCSNSOM to that of confocal FCS. In order to do this, we have measured the dependence of the FCS-NSOM brightness per molecule and diffusion time on the laser excitation intensity over the limited range of intensities that can be used without causing damage to the probe aperture. This experiment indicates that photobleaching is not an issue at the highest powers used for FCS-NSOM, as no decrease in the residence time is observed with increasing excitation intensity (cf. supplementary material). Furthermore, matching the triplet state photophysics observed for OG in the FCS-NSOM experiments with that observed in confocal FCS measurements indicates that the brightness per molecule is roughly ten times higher for confocal than for NSOM at the same excitation intensity. Optimization of the NSOM probe design to allow higher excitation intensities and of the light collection efficiency by adding a higher NA objective and a pinhole to block out-of-focus light will be required to improve the efficiency of our current FCS-NSOM setup.

In summary, we demonstrate the application of FCS with near-field optical probes to achieve an observation area that is an order of magnitude below the diffraction limit. We show that Eq. (3) is a good approximation of the correlation functions expected from 2D diffusion with different step function observation profiles and that it fits the experimental diffusion correlation data very well. Using smaller apertures, it may be possible to achieve an additional order of magnitude reduction in the observation area. This will enable the study of membranes with higher concentrations of fluorophores than is currently possible with diffraction-limited techniques. FCS-NSOM with small apertures may prove useful for observations on cellular membranes, which possess submicron features such as lipid domains and macromolecular assemblies, because the small axial extent of the nearfield will minimize excitation of autofluorescence from the cytoplasm. FCS-NSOM also has potential to be used for measurements in solution and in two-color FCS applications where high concentrations of solutes are often required.

This work was supported in part by the Canadian Institute for Photonic Innovations (CIPI). We thank CIPI team members (Dr. P. Norton, Dr. N. Petersen, and Dr. D. Cramb) for useful discussions.

${ }^{1}$ L. Kastrup, H. Blom, C. Eggeling, and S. W. Hell, Phys. Rev. Lett. 94, 178104 (2005).

${ }^{2}$ J. Ries, T. Ruckstuhl, D. Verdes, and P. Schwille, Biophys. J. 94, 221 (2008).

${ }^{3}$ M. Leutenegger, M. Gösch, A. Perentes, P. Hoffmann, O. J. Martin, and T. Lasser, Opt. Express 14, 956 (2006).

${ }^{4}$ J. Wenger, P.-F. Lenne, E. Popov, H. Rigneault, J. Dintinger, and T. Ebbesen, Opt. Express 13, 7035 (2005).

${ }^{5}$ M. J. Levene, J. Korlach, S. W. Turner, M. Foquet, H. G. Craighead, and W. W. Webb, Science 299, 682 (2003).

${ }^{6}$ K. T. Samiee, J. M. Moran-Mirabal, Y. K. Cheung, and H. C. Craighead, Biophys. J. 90, 3288 (2006).

${ }^{7}$ K. Hassler, T. Anhut, R. Rigler, M. Gosch, and T. Lasser, Biophys. J. 89, L01 (2005)

${ }^{8}$ A. Ianoul, M. Street, D. Grant, J. Pezacki, R. S. Taylor, and L. J. Johnston, Biophys. J. 87, 3525 (2004).

${ }^{9}$ See EPAPS Document No. E-APPLAB-93-060841 for details of the confocal and FCS-NSOM experiments and modeling of the correlation functions. For more information on EPAPS, see http://www.aip.org/pubservs/ epaps.html.

${ }^{10}$ M. Przybylo, J. Sykora, J. Humpolickova, A. Benda, A. Zan, and M. Hof, Langmuir 22, 9096 (2006).

${ }^{11}$ J. A. Veerman, M. F. Garcia-Parajo, L. Kuipers, and N. F. Van Hulst, J. Microsc. 194, 477 (1999).

${ }^{12}$ J. A. Dix, E. F. Y. Hom, and A. S. Verkman, J. Phys. Chem. B 110, 1896 (2006).

${ }^{13}$ N. L. Thompson, T. P. Burghardt, and D. Axelrod, Biophys. J. 33, 435 (1981). 